\title{
Sur le traitement des saumures de fromagerie par les rayons ultraviolets
}

\author{
par \\ J. CASALIS - F.M. LUQUET et F. ROSSIER \\ Laboratoire d'Industrie Laitière de Douai
}

Au cours du séjour des fromages en saumure, il se produit entre la saumure et les fromages des échanges qui, en ce qui concerne les saumures, ont pour résultat :

$1^{\text {o }}$ une diminution progressive de leur concentration en sel, cette diminution provenant à la fois de la pénétration du sel dans les fromages, et de la dilution de la saumure par le sérum qui exsude des fromages;

$2^{\circ}$ une augmentation de leur teneur en azote protéique, que celui-ci provienne des protéines en solution dans le sérum (albumine et globuline), ou des fragments qui sont détachés des fromages au cours des manipulations auxquelles ces derniers sont soumis; lactique.

$3^{\circ}$ une augmentation de leur teneur en lactose, et en acide

Ainsi la saumure s'enrichit-elle plus ou moins rapidement, suivant le type de fromages, en éléments qui peuvent servir d'aliments aux espèces microbiennes les plus diverses, toujours présentes sur les parois des bacs à saumure, sur la croûte des fromages et qui peuvent migrer avec le lactosérum de l'intérieur du fromage vers le bain de saumure.

Dans ces conditions, aux modifications de la composition chimique des saumures, vient s'ajouter une modification de leur flore microbienne. La population microbienne des bains de saumure, même si ceux-ci sont maintenus à une température relativement basse, peut augmenter rapidement, et provoquer:

- un abaissement du $\mathrm{pH}$ des saumures,

- une altération de leur odeur et de leur goût,

et par voie de conséquence, une modification de la flore microbienne sur la croûte et à l'intérieur des fromages, et une modification des qualités organoleptiques des fromages par pénétration à l'intérieur des fromages, des produits de dégradation des substances fermentescibles qu'elles contiennent. 
Pour limiter les risques que fait courir à la qualité des fromages la pollution microbienne des saumures, l'une ou l'autre des méthodes suivantes sont généralement employées :

a) soit le changement de la saumure lorsque l'acidité de cette dernière dépasse un seuil variable suivant les catégories de fromages :

- 220 Dornic pour les pâtes cuites,

- 50 Dornic pour les pâtes molles;

b) soit un traitement thermique de la saumure dans un échangeur à plaques, ou dans un échangeur tubulaire. II s'agit-là d'une pasteurisation qui a pour objectif de détruire le plus grand nombre possible de germes microbiens. Mais, ce traitement ne va pas sans difficulté. Les combinaisons classiques: temps $\mathrm{x}$ température de la pasteurisation, ne permettent pas la destruction des germes thermorésistants, et surtout des germes sporulés. D'autre part, sous l'effet de la chaleur, il se produit une floculation des matières protéiques, floculation qui peut encrasser les appareils, et gêner par la suite les échanges thermiques. Ce dernier inconvénient peut être considérablement réduit, en faisant précéder la pasteurisation, par une réduction de l'acidité grâce à l'addition de sels basiques (chaux en particulier). Cette désacidification est au reste nécessaire pour ramener la saumure au taux d'acidité favorable à des échanges normaux entre la saumure et les fromages.

\section{$* *$}

Nous avons appliqué aux saumures le traitement par les rayons ultraviolets que nous avons précédemment décrit à propos des eaux utilisées en industrie laitière (Cf. "LE LAIT ", nos 463-464, p. 157 à 166 ), et le présent mémoire résume le résultat de ces essais.

\section{I. - CONDITIONS GENERALES DES ESSAIS}

Les essais ont été effectués avec l'appareil DE STOUTZ 250 litres; heure décrit dans la publication ci-dessus mentionnée. Ils ont comporté deux séries d'expériences: l'une au laboratoire sur des quantités limitées de saumure, l'autre dans une importante fromagerie du département du Nord.

L'examen bactériologique des échantillons prélevés a été effectué en utilisant les méthodes suivantes :

- Coliformes : bouillon lactosé au vert brillant-désoxycholate-lactoseagar,

- Indologènes : eau peptonée,

- Streptocoques fécaux : test présomptif : milieu de ROTHE - Test confirmatif : milieu de LITSKY, 
- Clostridia sulfito-réducteurs : milieu V.L. au sulfite de $\mathrm{Na}$ à 0.25 p. 100 ,

- Clostridia protéolytiques: milieu Tryptone T. 65 ,

- Clostridia glucidolytiques: milieu Soytone S. 65 ,

- Flore totale aérobie: milieu Tryptone et Gélose nutritive,

- Flore anaérobie: milieu de ROSENOW cystéiné,

- Lactobacilles : milieu de MAN - ROGOSA et SHARPE,

- Streptocoques: milieu Yeast - Lemco - Agar,

- Levures et Moisissures : bouillon de haricots lactosé et gélosé à $\mathrm{pH} 3,5$.

\section{II. - ESSAIS AU LABORATOIRE}

\section{A) Essais en circuit ouvert}

Une première série d'essais a été faite, en faisant passer une seule fois dans l'appareil, de la saumure provenant d'une fromagerie fabriquant des fromages type Edam et Gouda. Le débit de l'appareil était de 235 litres/heure; la température de la saumure $19{ }^{\circ} \mathrm{C}$. Répétées à plusieurs reprises, ces expériences ont donné des résultats très voisins. Les tableaux I et II donnent les chiffres obtenus au cours de l'une d'entre elles.

Les conclusions de cette première série d'essais sont :

a) qu'à l'exception de la D.B.O., les caractéristiques physiques et chimiques de la saumure ne sont pas modifiées par le traitement aux ultraviolets. Les différences constatées dans les résultats avant et après traitement, sont dans les limites de précision des méthodes analytiques, et peuvent être aussi la conséquence d'un manque d'homogénéité de la saumure.

Pour ce qui est de la D.B.O., elle varie toujours dans le même sens, et augmente de 0,8 à $1,5 \mathrm{ppm}$ au cours du traitement ;

b) que la flore microbienne est plus ou moins complètement détruite, cette destruction étant particulièrement marquée en ce qui concerne la flore aérobie, les bacilles et streptocoques lactiques, les levures et moisissures. Pour les sporulés, cette première série d'essais ne peut être concluante, étant donné qu'à l'origine, la saumure n'en contenait qu'un nombre très faible.

\section{B) Essais en circuit fermé}

Dans une seconde série d'essais au laboratoire, un dispositif expérimental dont le schéma est donné à la figure 1 a permis d'étudier la marche en continu de l'appareil pendant 20 à 40 heures. Une augmentation progressive de la température de la saumure étant provoquée par le passage en continu à travers l'appareil, un dispositif de refroidissement a été intercalé dans le circuit. Le débit de l'appareil était de 235 litres/heure, la température de la saumure de $25^{\circ} \mathrm{C}$. 
TABLEAU. I. - Analyses physiques et chimiques d'une saumure de fromagerie avant et après traitement aux ultraviolets

\begin{tabular}{|c|c|c|}
\hline & $\begin{array}{l}\text { Saumure } \\
\text { avant } \\
\text { traitement }\end{array}$ & $\begin{array}{l}\text { Saumure } \\
\text { après } \\
\text { traitement }\end{array}$ \\
\hline $\mathrm{NaCl}$ en grammes par litre & 30.19 & 30.15 \\
\hline Cendres p. 100 & 16.08 & 14.94 \\
\hline Matière sèche totale p. 100 & 17.25 & 17.22 \\
\hline D.B.O. p.p.m. & 3 & 3.8 \\
\hline $\mathrm{pH}$ & 5.47 & 5.44 \\
\hline Acidité (en degré Dornic) & 24.5 & 24.5 \\
\hline
\end{tabular}

TABLEAU II. - Analyses bactériologiques d'une saumure de fromagerie avant et après traitement aux ultraviolets (nombre de germes par millilitre)

\begin{tabular}{|c|c|c|}
\hline & $\begin{array}{l}\text { Saumure } \\
\text { avant } \\
\text { traitement }\end{array}$ & $\begin{array}{l}\text { Saumure } \\
\text { après } \\
\text { traitement }\end{array}$ \\
\hline Flore aérobie psychrophile & 2518 & 202 \\
\hline Flore aérobie mésophile. & 36400 & 410 \\
\hline Coliformes & 10 & 0 \\
\hline Streptocoques fécaux & de 100 à 1000 & 0 \\
\hline Flore totale anaérobie & de 10 à 100 & 0 \\
\hline Clostridia protéolytiques & 27 & 0 \\
\hline Clostridia glucidolytiques .. & 0 & 0 \\
\hline Clostridia sulfito-réducteurs & 0 & 0 \\
\hline Bacilles lactiques & 288 & 1 \\
\hline Streptocoques lactiques & 8790 & 94 \\
\hline Levures et moisissures & 106 & 2 \\
\hline
\end{tabular}

Pour remplacer les germes microbiens détruits au cours de chaque passage, un réensemencement continu de la saumure a été fait au moyen d'une culture microbienne contenant en particulier, quelques-unes des espèces microbiennes les plus nuisibles en fromagerie.

Les caractéristiques de l'inoculum employé au cours de l'une des expériences sont données dans les tableaux III et IV. 
TABLEAU IV. - Population microbienne de l'inoculum

\begin{tabular}{|c|c|}
\hline $\begin{array}{c}\text { Germes microbiens } \\
\quad-\end{array}$ & Nombre par millilitre \\
\hline Coliformes $\ldots \ldots .$. & $10^{6}$ \\
\hline Streptocoques fécaux & $10^{6}$ \\
\hline Flore totale aérobie $\ldots \ldots \ldots$. & $10^{6}$ \\
\hline Clostridia protéolytiques $\ldots \ldots \ldots \ldots \ldots \ldots \ldots$ & $10^{7}$ \\
\hline Clostridia glucidolytiques $\ldots \ldots \ldots \ldots \ldots \ldots$ & $10^{7}$ \\
\hline Bacilles lactiques $\ldots \ldots \ldots \ldots \ldots \ldots \ldots \ldots \ldots \ldots \ldots \ldots \ldots$ & $8 \times 10^{6}$ \\
\hline Streptocoques lactiques ....... & $72 \times 10^{6}$ \\
\hline Levures et moisissures $\ldots \ldots \ldots \ldots \ldots \ldots \ldots$ & $27 \times 10^{3}$ \\
\hline
\end{tabular}

L'inoculum est incorporé à la saumure grâce à un goutte-à-goutte qui laisse tomber 4 gouttes par minute, ce qui représente une dose de 1/10 de $\mathrm{ml}$ d'inoculum par litre de saumure et par heure. Cet apport est largement supérieur à la population microbienne normale des saumures de fromagerie.

Les tableaux V et VI donnent les résultats de l'un des essais effectués avec de la saumure ensemencée au moyen de l'inoculum.

Cette deuxième série d'essais montre:

a) qu'à l'exception de la D.B.O. et du $\mathrm{pH}$, les caractéristiques physiques et chimiques de la saumure ne varient pas sensiblement au cours de l'expérience. La D.B.O. augmente au fur et à mesure du passage de la saumure dans l'appareil. Le $\mathrm{pH}$ augmente lui aussi mais de façon moins significative;

b) que les conclusions de l'essai en circuit ouvert sont confirmées en ce qui concerne la destruction de la flore microbienne. Cependant il semble que la destruction soit inversement proportionnelle à la population initiale.

$\mathrm{Au}$ bout de 24 heures de marche continue, le traitement est toujours efficace. Au bout de 40 heures, l'effet bactéricide diminue, car il se forme progressivement sur les parois internes des tubes de quartz de l'appareil, un dépôt principalement constitué de cristaux de chlorure de sodium qui gêne la pénétration des rayons ultraviolets dans la saumure.

\section{III. - ESSAIS PRATIQUES EN USINE}

Pour vérifier dans les conditions de la pratique, les résultats obtenus au Laboratoire, des essais ont été effectués au moyen de l'appareil précédemment décrit, dans une importante fromagerie de la région du Nord fabriquant des fromages du type Edam, Gouda et Saint-Paulin. 
TABLEAU V. - Analyse chimique d'une saumure traitée en circuit fermé par les rayons ultraviolets

\begin{tabular}{|c|c|c|c|c|c|c|}
\hline & $\begin{array}{c}\text { avant } \\
\text { traitement }\end{array}$ & $\begin{array}{c}\text { après } \\
4 \text { heures }\end{array}$ & $\begin{array}{c}\text { après } \\
8 \text { heures }\end{array}$ & $\begin{array}{c}\text { après } \\
12 \text { heures }\end{array}$ & $\begin{array}{c}\text { après } \\
16 \text { heures }\end{array}$ & $\begin{array}{c}\text { après } \\
20 \text { heures }\end{array}$ \\
\hline $\mathrm{NaCl}$ en gramme par 1. & 31.59 & 31.59 & 33.93 & 30.42 & 31.59 & 30.42 \\
\hline Cendres p. 100 & 18.45 & 17.62 & 17.39 & 17.92 & 17.23 & 19.32 \\
\hline M.S.T. p. 100 & 19.54 & 19.25 & 19.40 & 19.21 & 19.35 & 21.07 \\
\hline D.B.O. $\mathrm{ppm}$ & 1.5 & 1.8 & 2.3 & 2.7 & 2.9 & 3.5 \\
\hline $\mathrm{pH}$ & 5.57 & 5.58 & 5.57 & 5.49 & 5.57 & 5.60 \\
\hline Acidité en degrés Dornic & 24 & 24 & 23.5 & 24 & 23,5 & 24 \\
\hline
\end{tabular}


TABLEAU VI. - Analyse bactériologique d'une saumure traitée en circuit fermé par les rayons ultraviolets (nombre de germes par millilitre)

\begin{tabular}{|c|c|c|c|c|c|c|}
\hline Germes & $\begin{array}{c}\text { avant } \\
\text { traitement }\end{array}$ & $\begin{array}{l}\text { après } \\
4 \text { heures }\end{array}$ & $\begin{array}{l}\text { après } \\
8 \text { heures }\end{array}$ & $\begin{array}{c}\text { après } \\
12 \text { heures }\end{array}$ & $\begin{array}{c}\text { après } \\
16 \text { heures }\end{array}$ & $\begin{array}{c}\text { après } \\
20 \text { heures }\end{array}$ \\
\hline Flore aérobie psychrophile & 22600 & 43 & 234 & 19 & 65 & 45 \\
\hline Flore aérobie mésophile .. & 32600 & 17 & 1202 & 752 & 56 & 1000 \\
\hline Coliformes $\ldots \ldots \ldots \ldots \ldots$ & de 10 à 100 & 0 & 0 & 0 & 0 & 0 \\
\hline Streptocoques fécaux .... & de 10 à 100 & 0 & 0 & 0 & 0 & 0 \\
\hline Flore totale anaérobie .... & de 1000 à 10000 & de 100 à 1000 & de 100 à 1000 & de 100 à 1000 & 10 & 10 \\
\hline Clostridia protéolytiques .. & 25 & 0 & 1 & 0 & 2 & 2 \\
\hline Clostridia glucidolytiques & 10 & 33 & 2 & 0 & 0 & 6 \\
\hline Bacilles lactiques .... & 130 & 0 & 0 & 0 & 0 & 0 \\
\hline Streptocoques lactiques .. & 22750 & 33 & 33 & 34 & 805 & 5 \\
\hline Levures et moisissures ..... & 27050 & 5 & 113 & 11 & 276 & 70 \\
\hline
\end{tabular}


Le dispositif expérimental est représenté par le schéma de la figure 2: la saumure à traiter est filtrée puis pompée dans un bac tampon avant de passer dans l'appareil d'irradiation.

\section{FIGURE 2}

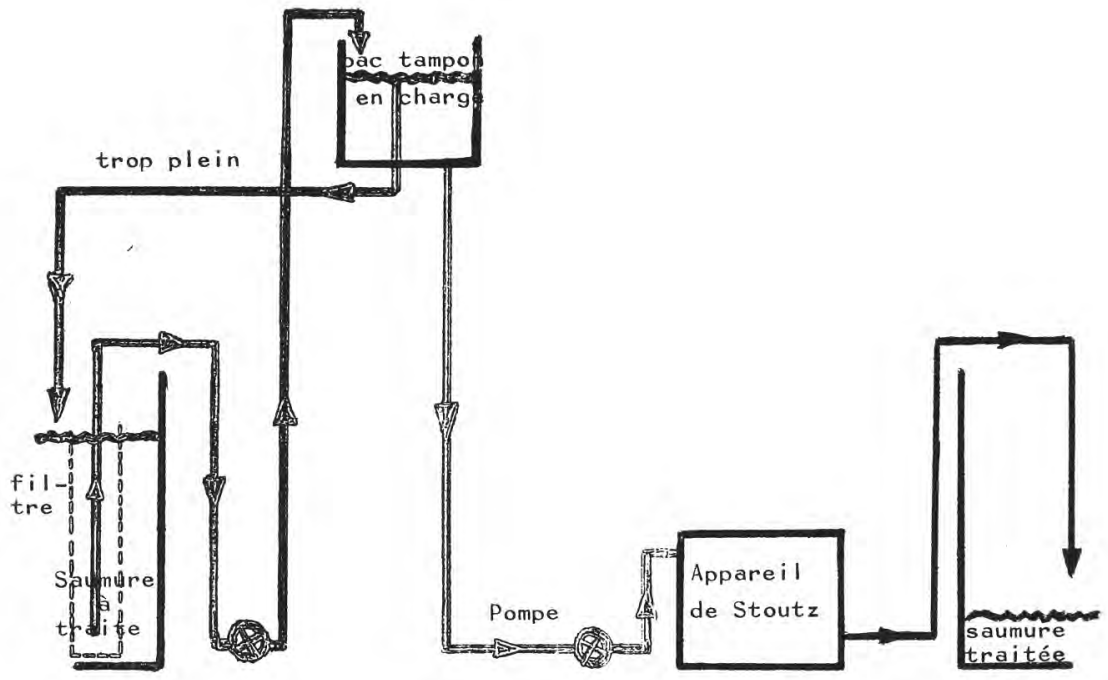

Le débit de l'appareil est de 220 litres/heure et la température de $23^{\circ} \mathrm{C}$.

Les tableaux VII et VIII donnent les principaux résultats de l'une des expériences qui ont été effectuées, au cours d'un fonctionnement de l'appareil pendant 71 heures. Les échantillons étaient prélevés toutes les cinq heures, mais pour alléger les tableaux, il ne sera fait mention que de prélèvements espacés de 24 heures.

L'examen des tableaux VII et VIII et les nombreux résultats analytiques rassemblés, qu'il ne paraît pas nécessaire d'inclure dans cet article, montrent que les essais en usine confirment les essais de Laboratoire. En faisant fonctionner l'appareil en circuit ouvert :

a) on ne modifie pas les caractéristiques physiques et chimiques de la saumure, exception faite pour la D.B.O. qui augmente de quelques dixièmes au cours du traitement;

b) la flore microbienne subit une destruction importante dont le pourcentage néanmoins varie suivant les espèces. Le classement de 
TABLEAU VII. - Caractéristiques physiques et chimiques d'échantillons de saumure

\begin{tabular}{|c|c|c|c|c|}
\hline . & avant & $\begin{array}{l}\text { après } 24 \text { heures } \\
\text { de fonctionnement }\end{array}$ & $\begin{array}{l}\text { après } \\
45 \text { heures }\end{array}$ & $\begin{array}{l}\text { après } \\
68 \text { heures }\end{array}$ \\
\hline $\mathrm{NaCl}$ en $\mathrm{g}$ par litre $\ldots . .$. & 23.42 & 23.35 & 23.46 & 23.40 \\
\hline Cendres p. $100 \ldots \ldots$ & 15.92 & 15.85 & 15.93 & 16.01 \\
\hline Matière sèche totale p. $100 \ldots \ldots \ldots \ldots$ & 18.51 & 18.22 & 18.27 & 17.92 \\
\hline D.B.O. $\operatorname{ppm} \ldots \ldots \ldots \ldots \ldots \ldots \ldots \ldots$ & 1.5 & 1.7 & 1.9 & 1.8 \\
\hline $\mathrm{pH} \quad \ldots .$. & 5.3 & 5.2 & 5.2 & 5.3 \\
\hline Acidité en degrés Dornic .. & 30 & 30 & 31 & 30 \\
\hline
\end{tabular}


TABLEAU VIII. - Population microbienne des saumures (nombre de germes par millilitre)

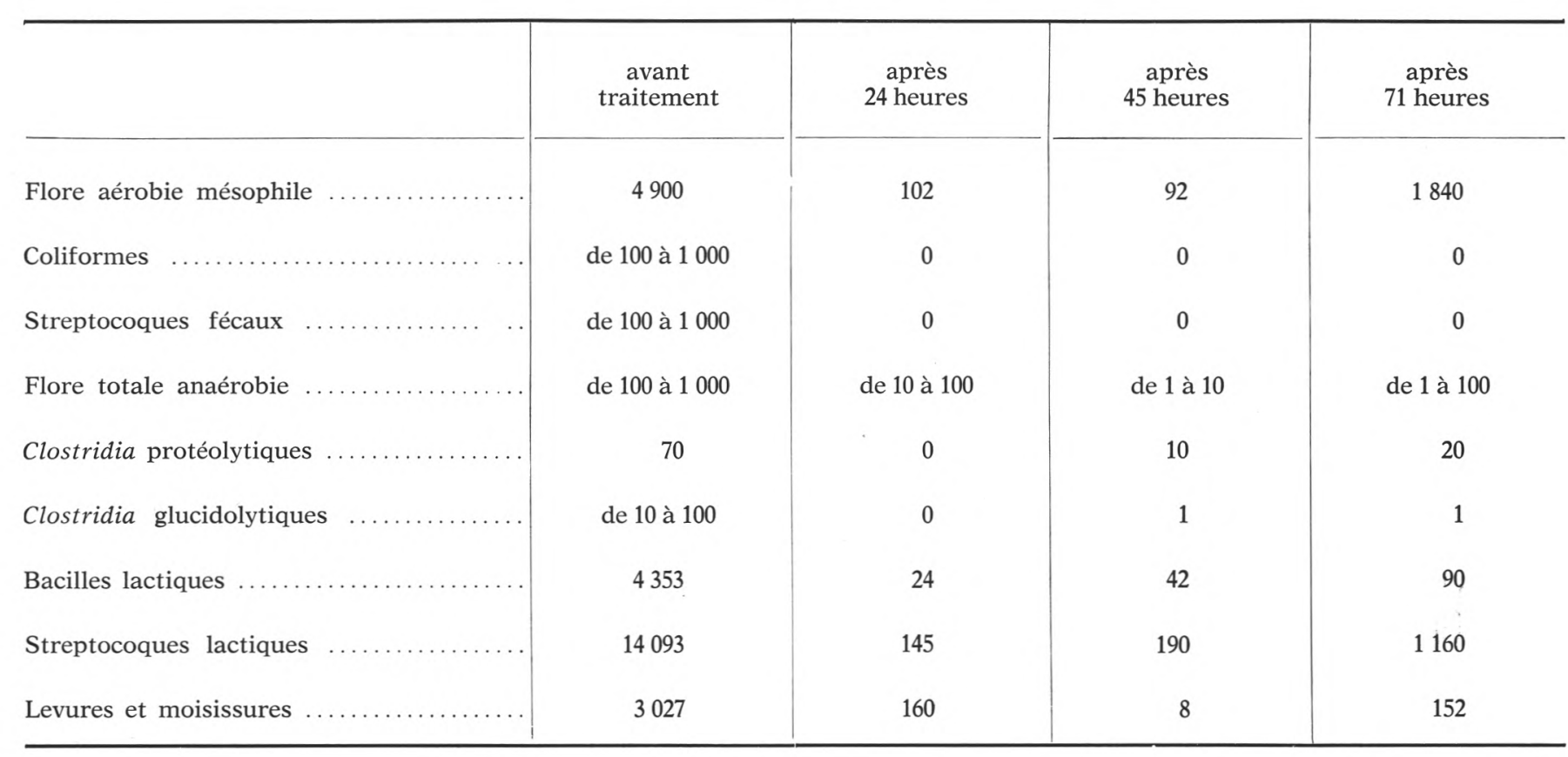


ces dernières en fonction de leur sensibilité décroissante aux effets microbicides des rayons ultraviolets est le suivant:

- coliformes, streptocoques fécaux, flore psychrophile et mésophile, bacilles lactiques, streptocoques lactiques, levures et moisissures, clostridia.

\section{$R$ é $s$ u $\mathrm{m}$ é}

Appliqué aux saumures de fromagerie, le traitement par les ultraviolets, décrit à propos des eaux utilisées en industrie laitière (Cf. «LE LAIT », nos 463-464, p. 157 à 166) donne les résultats suivants :

Les caractéristiques physiques et chimiques de la saumure ne sont pas modifiées.

La flore microbienne subit une destruction très importante, dont le pourcentage est néanmoins variable selon les espèces microbiennes considérées.

\section{S u m m a r y}

Ultraviolet treatment for water used in the dairy industry (see "LE LAIT », nos 463-464, pages 157 à 166) applied to brines used in cheese making gives results as below :

The physical and chemical characteristics of the brine are not affected.

The microbial flora is destructed to a large extent, but this extent is related to the microbial species concerned.

Reçu pour publication en octobre 1968. 